\title{
MAGNETIC-FIELD-DEPENDENT RECOMBINATION KINETICS OF GEMINATE RADICAL PAIRS IN REVERSED MICELLES OF VARIABLE SIZE
}

\author{
Thomas ULRICH and Ulrich E. STEINER \\ Fakultät für Chemie, D-7750 Konstanz, Federal Republic of Germany
}

\begin{abstract}
The magnetic-field-dependent kinetics of geminate radical pairs, created by electron transfer from aniline io thionine triplet in reversed micelles has been studied by nanosecond laser flash spectroscopy. Depending on the water concentration, the rate constant of intramicellar recombination is reduced by a factor between 2.4 and 3.2 in a field of $1 \mathrm{~T}$. The $B_{1 / 2}$ values increase from about 15 to $48 \mathrm{mT}$ when the water-pool radius is increased by a factor of 4 . The experimental results demonstrate that triplet-singlet transitions cannot be rate determining in zero field. However, $T_{ \pm} \rightarrow T_{0}$ and $T_{ \pm} \rightarrow S$ radical pair spin relaxation must become rate determining as the magnetic field is increased above $10 \mathrm{~m} T$.
\end{abstract}

\section{Introduction}

When radical pairs (RPs) are produced within micelles the time period of geminate re-encounters is in the microsecond region, which is longer by two or three orders of magnitude than in homogeneous solutions. Thus the micellar supercage effect as first applied by Turro and co-workers $[1,2]$ to increase the magnetic field sensitivity of $R P$ reactions, should be considered as an attractive starting point for experimental and theoretical studies of the long-time behaviour of spincorrelated pairs.

As we have pointed out [3] recent work on magnetic-field-dependent kinetics of triplet spin correlated RPs in micelles reveals rather common features:

(i) the ratc constant of intramicellar pair recombination is of the order of some $10^{6} \mathrm{~s}^{-1}$ irrespective of the chemical nature of the radicals;

(ii) the magnetic field dependence does not saturate at low field strengths, typical for the isotropic hyperfine coupling ( $\mathrm{hfc}$ ) of the respective pairs, but seems to extend to field strengths, as high as $1 \mathrm{~T}$.

As we have discussed [3], there anise serious problems if one tries to explain the intramicellar recombi- . nation rate constant and its magnetic field dependence in terms of the usual RP mechanism [4], where coherent spin motion due to the isotropic hfc determines the rate of formation of cage from triplet RPs.

Recently Hayashi et al. $[5,6]$ suggested that the spin relaxation mechanism, as originally conceived by Brockehurst [7], accounts for the observed RP kinetics in micelles under high magnetic fields. In this paper we present a study of the magnetic-field-dependent recombination kinetics of RPs in reversed micelles, which has been investigated as a function of the intramicellar water-pool radius. Our results provide strong evidence that the realxation mechanism is of major importance under micellar conditions.

\section{Experimental}

The materials used were the same as specified previously [3]. Reversed micellar solutions were prepared by dissolving cetyldimethylbenzylammonium chloride (CDBA) $0.04 \mathrm{M}$ in benzene. Thionine was solubilized with a concentration of $5 \times 10^{-6} \mathrm{M}$ by adding suitable amounts of corresponding aqueous stock solutions, allowing for variable concentration of water. Before the investigation the solutions were flushed with nitrogen (oxygen content less than $5 \mathrm{ppm}$ ) for $30 \mathrm{~min}$.

The kinetic investigations were performed with a nanosecond laser flash spectrometer to be described in more detail in a forthcoming paper [8]. Thionine 
was excited by a dye laser pulse (rhodamine 6G, 575 $\mathrm{nm}$ ) in a flow cuvette of $1 \mathrm{~cm}$ pathlength. The flashed solution was replaced after each laser pulse. Each transient signal was averaged over 64 pulses. The time resolution was determined by the laser pulse duration of about $15 \mathrm{~ns}$.

\section{Results and discussion}

As described previously, thionine $\left(\mathrm{TH}^{+}\right)$may be solubilized in the water pool of reversed micelles of CDBA in benzene and after optical excitation reacts in its triplet state with aniline to produce intramicellas RPs via electron transfer from aniline to thionine triplet (cf. fig. 1). By using suitable water concentrations the size of the intramicellar water pools can be varied in a defined manner. For AOT rcversed micelles it has been established by several independent methods $[9,10]$, that above a certain water concentration the radius of the water pool varies linearly with the molar ratio $c^{\prime}=c_{\mathrm{H}_{2} \mathrm{O}} / c_{\text {surfactant }}$. For our CDBA/benzene reversed micellar system this linear relation has been found to hold approximately between $c^{\prime}=10$ and $c^{\prime}=32[11]$, above which phase separation occurs. Observing the decay of the triplet absorption of $\mathrm{TH}^{+}$ at $780 \mathrm{~nm}$ we find dynamic triplet quenching by aniline up to a quencher concentration of $0.1 \mathrm{M}$. In order to minimize excited singlet state quenching our studies were performed with an aniline concentration of 0.05 M. With this quencher concentration the lifetime of ${ }^{3} \mathrm{TH}^{+}$amounted to $15 \mathrm{~ns}$ for $c^{\prime}=8$ and 30 ns for $c^{\prime}$ $=32$.

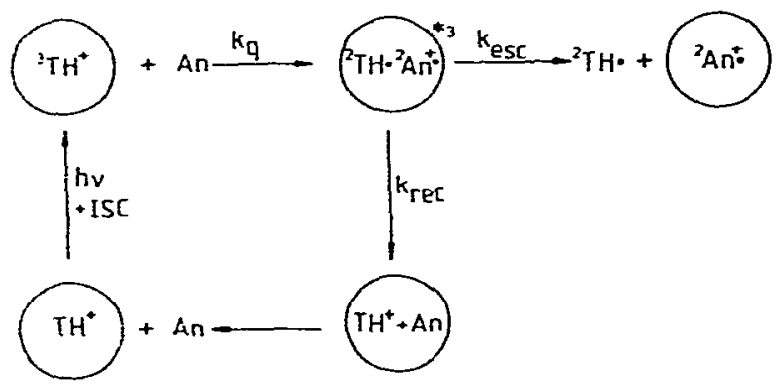

Fig. 1. Reaction scheme for thionine triplet $\left({ }^{3} \mathrm{TH}^{+}\right)$and aniline (An) in reversed micellar solution. The circles indicate the water poul of the reversed micelles. The symbol $* 3$ denotes that the RP is created with parallel spins.
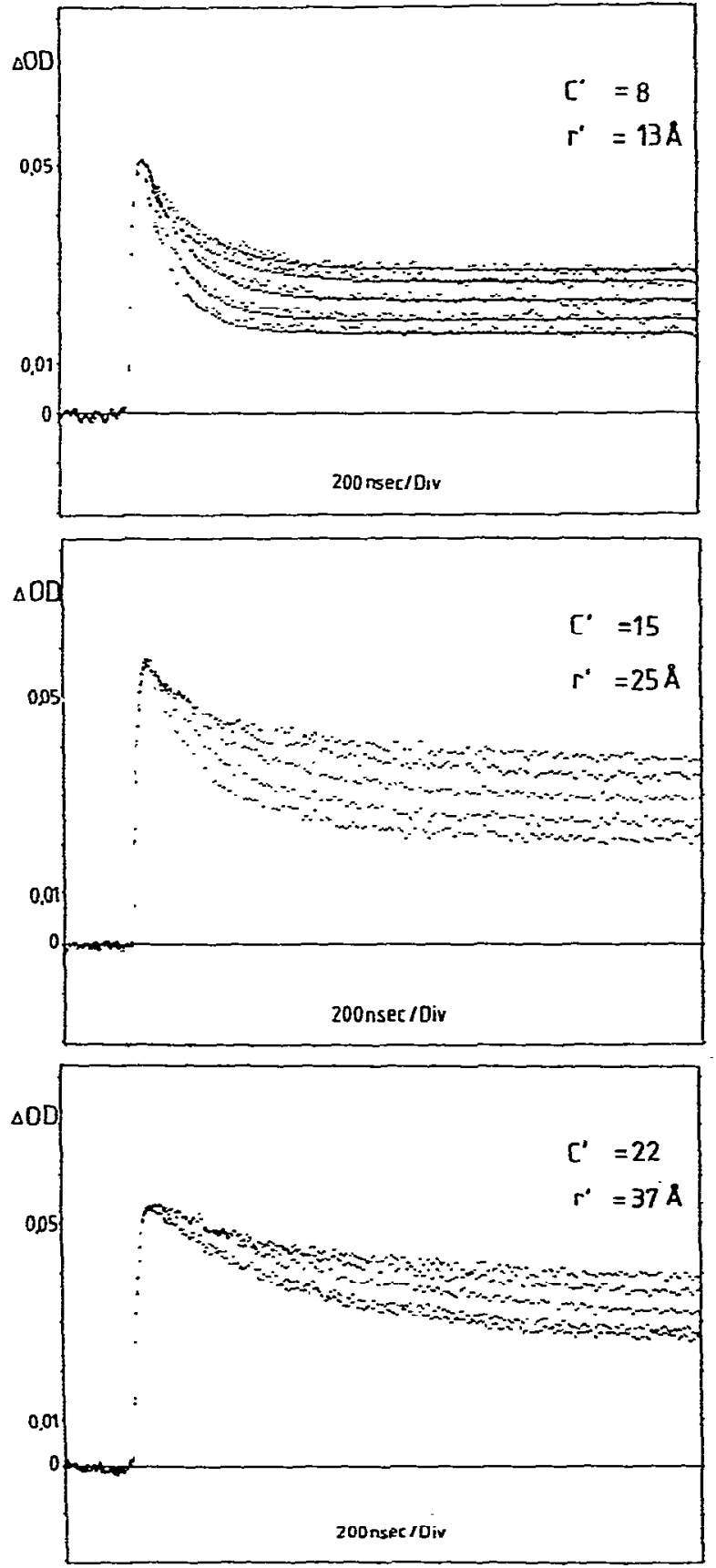

Fig. 2. Decay kinetics of the Iadical TH', observed as transient absorption at $\mathbf{4 2 5} \mathrm{nm}$ for various magnetic fiejds (in each graph from below): $0,5,20,100,1000 \mathrm{mT}$. The water concentrations $c^{\prime}$ are indicated together with the corresponding water-pool radii. The solid curves for $c^{\prime}=8$ are best fits using eq. (1). 
The decay kinetics of the radicals produced in the triplet reaction may be conveniently followed at 425 $\mathrm{nm}$ (absorption of semithionine $\mathrm{TH}^{-}$) or at $610 \mathrm{~nm}$ the wavelength of maximum ground state absorption. The kinetics at both wavclengths is the same, indicating that disappearence of thionine radicals is in fact due to electron back-transfer with repopulation of the dye ground state.

In fig. 2 are shown transient absorption signals monitoring the decay of thionine radicals. The decay occurs in two steps. The fast decay, which we interpret as intramicellar geminate radical pair recombination, is completed within 200 to $2000 \mathrm{~ns}$ depending on the size of the water pools and the strength of an external magnetic field. The second stage of radical recombination, probably a second-order homogeneous process, is much slower and not time resolved in our experiments. As demonstrated by the signals in fig. 2, the fast radical decay is considerably slowed down by the influence of an external magnetic field and also by an increase of the water pool radius. The kinetics of the fast radical decay is satisfactorily described by a competition of two first-order processes(fig. 1) leading either to intramicellar pair recombination $\left(k_{\text {rec }}\right)$ or to escape $\left(k_{\text {esc }}\right)$ of one of the radicals (probably the neutral dye semiquinone radical $\mathrm{TH}^{*}$ ) from the micelle. The corresponding time dependence is given by

$$
\begin{aligned}
& c_{\mathrm{TH}} \cdot(t)=c_{\mathrm{TH}} \cdot(0)\left[k_{\mathrm{esc}} /\left(k_{\mathrm{esc}}+k_{\mathrm{rec}}\right)\right] \\
& \quad \times\left\{1+\left(k_{\mathrm{rec}} / k_{\mathrm{ecs}}\right) \exp \left[-\left(k_{\mathrm{esc}}+k_{\mathrm{rec}}\right) t\right]\right\},
\end{aligned}
$$

which has been used to simulate the decay curves numerically and thus extract the rate constants $k_{\text {rec }}$ and $\overline{k_{\text {esc }}}$ as a function of the magnetic field and the water-pool radius. The ratio $k_{\text {esc }} /\left(k_{\text {rec }}+k_{\text {esc }}\right)$ represents the fraction $\Phi_{\text {esc }}$ of escape radicals which we detccted previously in our microsecond time-rcsolved experiments [3]. The present results are consistent with our previous ones.

The variation of rate constants $k_{\text {esc }}$ and $k_{\text {rec }}$ with the radius of the water pool as observed in zero magnetic field is depicted in fig. 3 . The variation of both rate constants is centered at about $10^{6} \mathrm{~s}^{-1}$, however $k_{\text {rec }}$ is much more sensitive to variations of the waterpool radius than $k_{\text {esc }}$-Using double-log plots we obtain straight lines (at least for $c^{\prime}>15$ where $c^{\prime}$ should be proportional to $r^{\prime}$ ) with slopes -3 for $k_{\text {TeC }}$ and -1.2 -for $k_{\text {esc }}$, i.e. $k_{\text {rec }}$ is proportional to $r^{\prime}-\overline{3}$ and $k_{\text {esc }}$ ap-

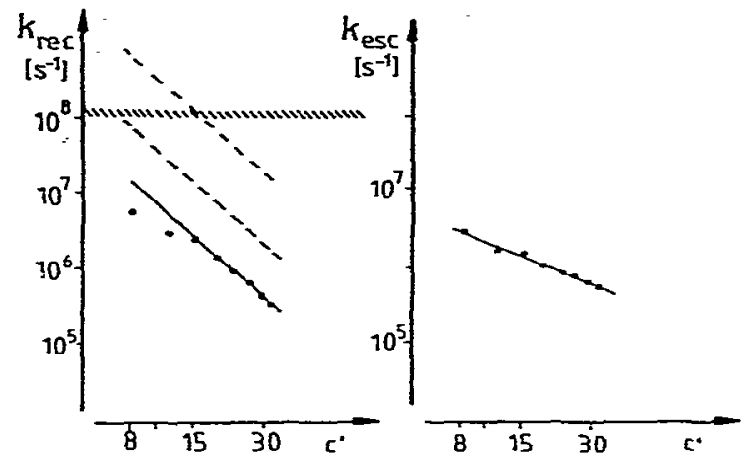

Fig. 3. Variation of rate constants $k_{\text {Iec }}$ and $k_{\text {esc }}$ (zero-field values) as a function of water concentration. The dashed lines (left) are theoretical border cases (eq.(2)) for the diffusion controlled intramicellar recombination.

proximately proportional to $r^{\prime-1}$.

Whereas the rate constant of escape does not show a significant magnetic field dependence, the recombination rate constant is reduced by a factor of almost three when increasing the field to $1 \mathrm{~T}$. The magnetic field effect on $k_{\text {rec }}$ is displayed in fig. 4 for various water concentrations, i.e. various radii of the micellar water pools. The magnetic field, where $k_{\text {rec }}$ has dropped by one half of the difference between zero field and $1 \mathrm{~T}$ is marked as $B_{1 / 2}$. Although there is considerable scatter in these values, it is evident that the $B_{1 / 2}$ values increase from about $13 \mathrm{mT}$ at $c^{\prime}=8$ to about $48 \mathrm{mT}$ at $c^{\prime}=32$.

Triplet-singlet spin conversion, diffusion and recombination rate constant. Since the RPs are created with triplet spin alignment, a triplet-singlet transition is necessary before recombination to singlet ground state products can occur. Assuming that a singlet RP would react with a probability $f$ in each encounter we may write for the rate constant of recombination:

$k_{\mathrm{rec}}=f p_{\mathrm{s}} k_{\mathrm{diff}}$,

where $\bar{p}_{s}$ denotes the average singlet probability acquired by the radical pair during the time intervals between encounters within the water pool and $k_{\text {diff }}$ is the (first-order) rate constant of intramicellar encounters. We have calculated the spin evolution resulting from the isotropic $h f c$ for the RP thionine-aniline [3]. According to this calculation we expect that a quasi-statistical electron spin alignment with 22\% 


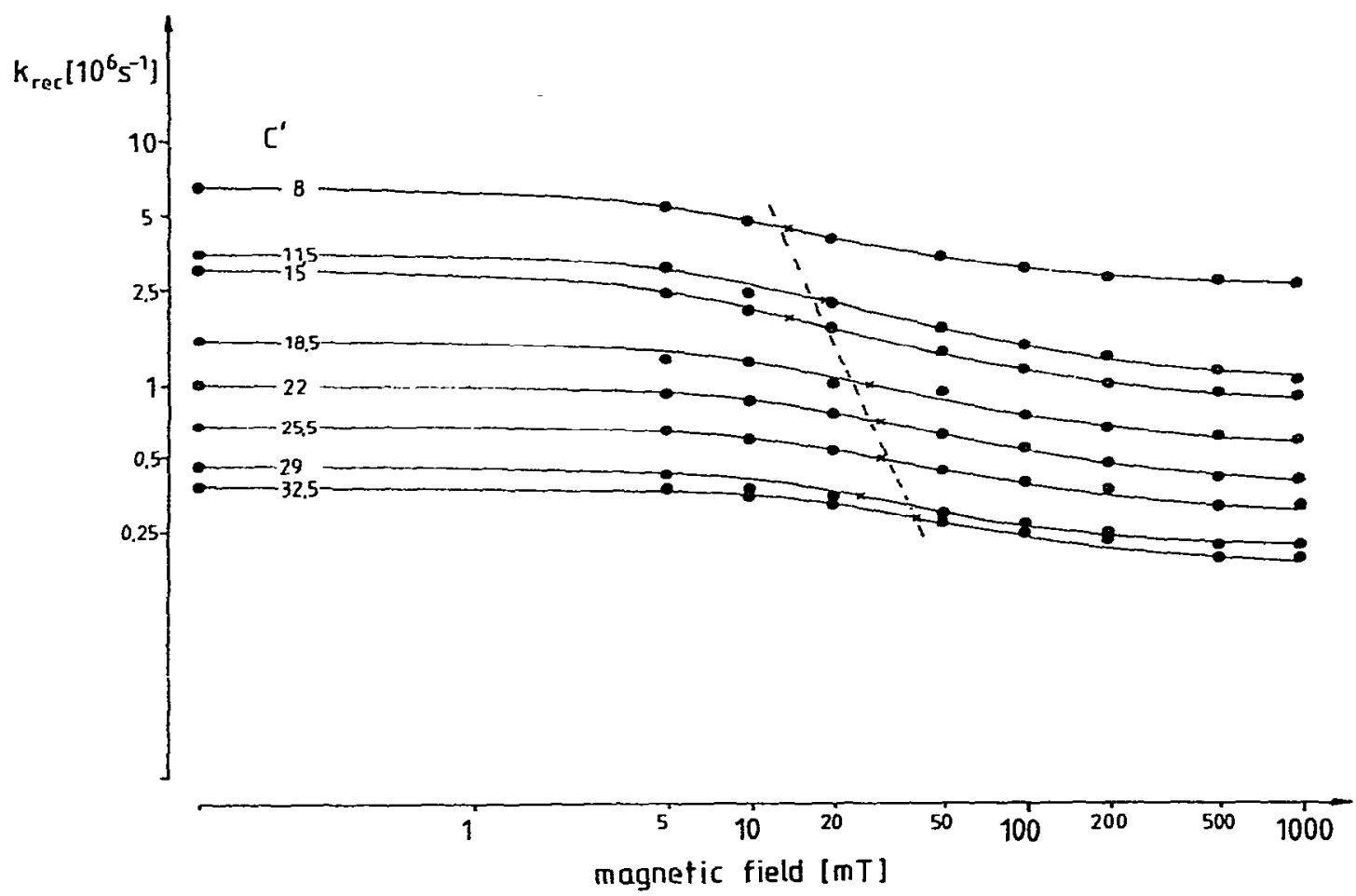

1'ig. 4. Magnetic field dependence of intramicellar radical recombination rate constant $k_{\text {rec }}$ for various water concentrations $c^{\prime}$ as indicated in the figure. $\bullet$ experimental points, $\mathrm{X}$ interpolated $B_{1 / 2}$ positions.

siuglet character is attained after about 10 ns. A corresponding rate constant of $10^{8} \mathrm{~s}^{-1}$ has been indicated as a reference line in fig. 3. On the other hand $k_{\text {diff }}$ may be estimated by eq. (3) (cf. refs. [12,13]), which is applicable if the water pool radius $r^{\prime}$ is several times larger than the encounter radius,

$k_{\text {Icc, diff }}=\left(2 R T / \pi \eta N_{\mathrm{L}}\right) r^{\prime-3}$.

Here $\eta$ is the viscosity of the intramicellar water pool. The other symbols have their usual meaning. So far, the int tamicellar water viscosity has not been unambiguously assessed in the literature. Although viscosities up to $100 \mathrm{cl}$ have been attributed to a thin shell of a few $A$ thickncss close to the water-surfactant interface $[14-16]$, the interior of the water pool for radii $r^{\prime}>15 \mathrm{~mm}$ is thought to be comparble in viscosity to bulk walcr. In fig. 3 are drawn reasonable border lines $(\eta=1 \mathrm{cl}$, (bulk water) and $\eta=10 \mathrm{cP}$ ) for the intrainleellut waler-poul viscosily. The figure demonstrates llat fin $c^{\prime}>15$ the time span $\tau_{\text {dirm }}$ between intramicel- lar reencounters $\left(\tau_{\text {diff }}=1 / k_{\text {diff }}\right)$ will be larger than the time required for achieving hfc-induced quasispin equilibrium (which is the opposite case to what we considered in our previous paper [3]). Therefore, at least for $c^{\prime}>15$ the $\mathrm{hfc}$-induced triplet-singlet transition cannot be the rate determining step for $\mathrm{RP}$ recombination. The recombination reaction for $c^{\prime}>15$ will be controlled by diffusion $\left(k_{\text {diff }}\right)$, spin statistics $\left(\bar{p}_{s}=1 / 4\right)$ and singlet pair reactivity $(f \leqslant 1)$. Since the latter quantities should not be $r$ dependent the $r^{\prime}-3$ dependence of $k_{\mathrm{fec}}$ will reflect the corresponding behaviour of $k_{\text {dirr }}$ (eq. (3)).

At a magnetic field of about $10 \mathrm{mT}$ the Zeeman splitting of the $T_{+}$and $T_{-}$level slould effectively prevent the spin conversion of these leyels as induced by the isotropic hfe, which is characterized by an effective value of $3.5 \mathrm{mT} \mathrm{[3].} \mathrm{Thus,} \mathrm{if} \mathrm{isotropic} \mathrm{hfc}$ alone were responsible for triplet-singlet conversion of the radical pair, at higher magnetic fields the kinetic behaviour of the RPs should become field indepen- 
dent, one third of them $\left(\mathrm{T}_{0}\right)$ recombining with a rate constant of $\frac{1}{2} f k_{\text {diff, the others }}\left(\mathrm{T}_{+}, \mathrm{T}_{-}\right)$, remaining unreactive. However, the observed behaviour is quite different. The fast recombination kinetics does not show significant deviation from an effective first-order process, its rate constant decreases with the magnetic field, showing a maior change between 10 and $50 \mathrm{mT}$ but is still not constant at $1 \mathrm{~T}$. From this we conclude that $T_{+}$and $T_{-}$RPs continue to contribute to tripletsinglet transitions even if the Zeeman splitting is much larger than the effective isotropic hfc $(3.5 \mathrm{mT})$. The mechanism for this transition can no longer be a coherent triplet-singlet coupling but must be identified with spin relaxation, brought about by anisotropic couplings and their stochastic modulation by the molecular tumbling motion.

The role of electron spin relaxation as a kinetic determinant for RP recombination has been first discussed by Brocklehurst [7]. More recently, Hayashi and Nakagura [6] have suggested the spin relaxation mechanism for explaining the effect of high magnetic fields on radical pair recombination in micelles. These authors analysed a kinetic situation (high-field case) where the triplet component $T_{0}$ is assumed to be in quasi-equilibrium with the singlet state (fast isotropic hyperfine mixing) and the triplet levels $T_{+}$and $T_{-}$ are kinetically connected to $T_{0}$ and $S$ via relaxation processes characterized by rate constants $k_{\mathrm{r}}$ and $k_{\mathrm{r}}^{\prime}$, respectively. If $k_{\mathrm{r}}+k_{\mathrm{r}}^{\prime}$ drops below the rate constant of product formation from $S$ (in our case assumed to equal $f k_{\text {diff }}$ ) the rate constant of recombination will be determinated by the rate constant of spin relaxation. In terms of spin resonance spectroscopy the relaxation under consideration is a $T_{1}$ process for which an expression of the form

$T_{1}^{-1}=\left\langle\omega_{a}^{2}\right\rangle \tau_{0}\left(1+\omega_{0}^{2} \tau_{0}^{2}\right)^{-1}$

has been derived [17]. Here $\omega_{0}$ is the Larmor frequen$c y, \tau_{0}$ the orientational correlation time of the radicals and $\left\langle\omega_{a}^{2}\right\rangle$ is a mean square frequency due to the anisotropic couplings familiar from ESR spectroscopy. The terms contributing to $\left\langle\omega_{a 1}^{2}\right\rangle$ are quadratic, linear or constant in the magnetic field. As shown in a model calculation by Hayashi and Nakagura [6] the contribution of the anisotropic hfc to $\left\langle\omega_{\mathrm{a}}^{2}\right\}$, which is independent of the external magnetic field may well provide the dominant contribution to the relaxation rate constant. If $\omega_{0}^{2} \tau_{0}^{2}>1$ the corresponding contribution to $T_{1}$ will be strongly quenched by the external magnetic field. It appears likely that this term could be responsible for the magnetic field dependence of $k_{\text {rec }}$ observed by us. Since the relaxation rate constant is reflected in the recombination rate constant only if the relaxation is slower than the recombination of a singlet $\mathrm{RP}$, which is proportional to $k_{\text {diff, it follows }}$ that higher magnetic fields will be necessary to meet this condition when the diffusion-controlled rate gets smaller, i.e. when the radius of the water pool increases. This relation would offer a resonable explanation for our observation that the half-field value increases with the radius of the water pool.

In concluding, we want to point out, however, that one main feature of the Hayashi-Nakagura model is not borne out in our observation. The kinetic model demands that the intramicellar decay kinetics of the RPs should become biexponential as the field increases. The recombination of $T_{0}$-born $R P s$ should show up in an exponential component with a rate constant of two times the zero-field rate constant. This fast component could not be resolved in our signals.

\section{Conclusion}

Although more experimental and theoretical work will be necessary for achieving a full understanding of the magnetic field effects on radical recombination in micelles, the present results strongly suggest that spin relaxation is of major importance on the time scale of intramicellar kinetics. We should like to point out, that in view of their long life time spin correlated RPs in micelles should represent excellent systems for observing microwave induced kinetic effects which so far have been detected only indirectly from stationary yield measurements (RYDMR) [18-20]. Thus the family of magnetic resonance methods might be supplemented by a further one which may be termed "reaction rate detected magnetic resonance". Reversed micelles with their easily adjustable size of the water core should be especially suited for exploiting a spectroscopy of this kind.

\section{Acknowledgement}

Financial support of this work by the Deutsche 
Forsehungsgemeingehart and by the Fonds der Chemischen Industrie is gratefully aeknowledged.

\section{References}

[1] N.J, Turro and B. Kraeutler. Acenunts Chem. Res. 13 (1980) 369 :

[2] $N, J, T u r r o$ and $G, C$, Weed,, Am, Cliem, Soe, 105 (1983) 1861,

[3] W. Schenker, Th. Ułfich and U,E, Steiner, Cliem, Phys, Letters 103 (1983) 118 ,

[4] K.M. Salikhov, Yu,N. Molin, R,Z, Sagdeev and A.L. Buchachenko, Spin polarisation and magnetic effects in radical retactions. Studies in physical and theoretical chemistry, Vol. 22 (Elsevier, Amsterdam, 1984).

[5] Y. Sakaguchi, H, Hayashi and S. Nagakura, J, Phys, Chem. 86 (1982) 3177;

Y. Sakaguchi and II. Llayashi, Chem. Pliys. Letters 87 (1982) 539.

[6] H. Hayashi and S. Nagakura, Bull, Chem. Spc, Japan 57 (1984) 322.

17] B. Brocklehurst, Nature 221 (1969) 921.
(8) Th, Ulfieh and $U, E_{2}$ Steinef, to be published,

[9] M, Zulauf and $F_{z}=I I$, Lieke, J, Phys, Chent 83 (1999) 480 ,

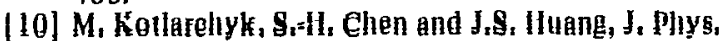
Cliem. 86 (1982) 3973.

[11] D. Millef, Disgeftation, Universität Stuttgaft (1977).

[1?] U, Gösele, U,K.A, Klein and M, Hauser, Chem, Phys, Letters $68(1979) 291$.

[13] M, D. Ilatlee, J,J, Kozak, G, Rothenberger, P., Infelta and M. Grätzel, J, Phys, Clıem. 84 (1980) 1508.

[14] G.D. Correll, R.N. Cheser, P. Nome and J.H. Pendler, J. Am, Chem, Soe, 100 (1998) 1254.

(15) Y,Y, Lim and J,H, Pendler, J, Am, Chem, Sog, 100 (1978) 7490,

[16] P.E. Zinsli, J, Phys, Chem, 83 (1979) 3223,

[17] A. Carrington and A.D. MeLachlan, Introduetion to magnetic resonance (Chapman and Hall, London, 1979),

[18] O,A, Anisimav, V,M. Grigeryants, V,K, Molchanov and Yu.N. Molin. Chem. Pliys, Letters E6 (1979) 265,

[19] J,R. Norris, M.K, Bowman, D,E, Budil, J, Tang, C,A, Wraight and G,L, Closs, Prof, Natl, Acad, Sci, US 79 (1982) 5532,

[20] S.I. Kubarev, S.V. Sheperstov and A,S, Shustoy, Chen, Phys. Letters 73 (1980) 370 , 